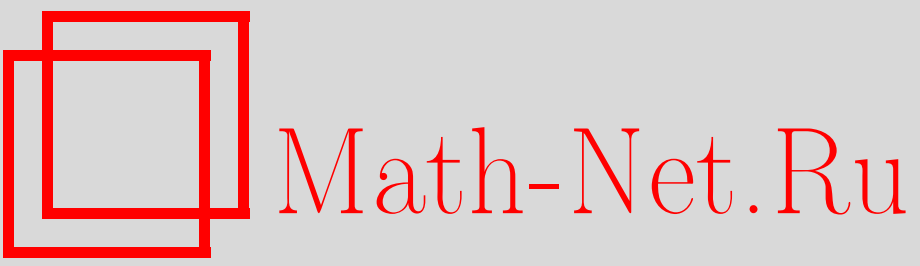

Н. И. Гоза, Алгебры с неприводимой немаксимальной группой автоморфизмов, УМН, 1996, том 51, выпуск 3, 193-194

DOI: https://doi.org/10.4213/rm980

Использование Общероссийского математического портала Math-Net.Ru подразумевает, что вы прочитали и согласны с пользовательским соглашением

http://www.mathnet.ru/rus/agreement

Параметры загрузки:

IP: 54.162 .127 .20

26 апреля 2023 г., 09:14:02 


\title{
АЛГЕБРЫ С НЕПРИВОДИМОЙ НЕМАКСИМАЛЬНОЙ ГРУППОЙ АВТОМОРФИЗМОВ
}

\author{
Н. И. ГОЗА
}

Рассмотрим линейную группу Ли $H$, действующую в линейном пространстве $L$ над полем $\mathbb{C}$, и ее линейное представление $\Phi$. Действие представления $\Phi$ может оказаться инвариантным, т.е. группа Ли $H$ может иметь тензоры, инвариантные относительно $\Phi$. Отметим, что чем шире группа $H$, тем размерность пространства ее инвариантных тензоров меньше. Если мы расштрим $H$ до линейной группы Ли $G$, заданной в том же пространстве $L$ и имеющей линейное представление $\Psi$, то, естественно, множество инвариантных тензоров не расширится при этом. Возникает вопрос, насколько изменится пространство инвариантных тензоров в таком случае? Задача эта весьма интересна, но для произвольных линейных групп Ли слишком обширна, поэтому ограничим ее каким-либо образом.

Софусом Ли классифицированы примитивные группы преобразований, действующие в пространствах одного, двух и трех измерений [1]. Проблема классификации равносильна задаче определения максимальных подгрупп в группах Ли (причем подгруппы не должны содержать нетривиальных нормальных делителей группы). Случай, когда группа не является простой, разобран В. В. Морозовым [2]. Проблема максимальных подгрупп простых групп решена Е. Б. Дынкиным в [3], где найдены максимальные подгруппы классических групп среди приводимых подгрупп, а также среди неприводимых подгрупп, не являющихся простыми. Наибольший интерес представляет случай простых неприводимых подгрупп.

В ходе сложного исследования Е.Б. Дынкиным был получен исключительный результат: в большинстве своем простые неприводимые группы Ли максимальны в одной из классических. То есть не являются частью никакой другой неприводимой группы, не совпадающей с классической. В силу этого класс простых неприводимых групп очень велик, что определенным образом затрудняет его изучение.

Вопрос о максимальных подгруппах классических групп непосредственно связан с другими очень интересными объектами - включениями между неприводимыми линейными группами Ли.

Теорема [3]. Полное перечисление всех типов включений $H \subset G$, əде $H$ и $G$-неприводимые группь унимодулярных преобразований, причем $G$ является простой и отлична от $\mathrm{SL}(N), \mathrm{Sp}(N)$ и $O(N)$, дается таблицей 5.

Приведенных в таблице "исключений Дынкина" всего 36 пар.

Учитывая все сказанное выше, мы остановимся на изучении пространств тензоров, инвариантных относительно представлений $\Phi(H)$ и $\Psi(G)$ неприводимых линейных групп Ли $H$ и $G$ включений $H \subset G$. Проследим изменение размерности таких пространств при расширении подгруппы $H$ до объемлющей группы $G$. Причем ограничимся тензорами третьей и четвертой валентности. так как, с одной стороны, на современном уровне развития теории инвариантов и теории представлений групп достаточно реально получить решение этой задачи, чего нельзя сказать о проблеме изучения инвариантных тензоров более высоких валентностей. С другой стороны, инвариантные тензоры третьей и четвертой валентности описывают многие интересные геометрические объекты.

Основные результаты работы приведены в следующей теореме.

Теорема. Пусть $H$ и $G$ - неприводимые группь унимодулярных линейных преобразований включения $H \subset G$, где $G$ - проста и не совпадает с $\operatorname{SL}(N), \operatorname{Sp}(N)$ и $\mathrm{O}(N)$. $\Phi$ и $\Psi$ - неприводимье представления групп $H$ и $G$, соответственно.

Размерность пространства тензоров $c_{j q}^{i}\left(c_{i j q}\right)$, инвариантных относительно $\Phi(H)$ $u \Psi(H)$, не меняется при расширении подгруппь $H$ до группь $G u$

а) равна 0 для следующих включений: $\mathrm{I}_{8}, \mathrm{I}_{9}, \mathrm{I}_{10}, \mathrm{I}_{12}, \mathrm{II}_{3}, \mathrm{II}_{5}, \mathrm{II}_{7}, \mathrm{II}_{9}, \mathrm{IV}_{2}, \mathrm{IV}_{3}, \mathrm{IV}_{4}$, $\mathrm{IV}_{5}, \mathrm{IV}_{6}, \mathrm{IV}_{8}, \mathrm{IV}_{9}\left(\mathrm{I}_{8}, \mathrm{I}_{9}, \mathrm{II}_{3}, \mathrm{II}_{5}, \mathrm{II}_{7}, \mathrm{II}_{9}, \mathrm{IV}_{2}, \mathrm{IV}_{3}, \mathrm{IV}_{4}, \mathrm{IV}_{5}, \mathrm{IV}_{6}, \mathrm{IV}_{8}, \mathrm{IV}_{9}\right)$;

б) равна 1 для включений $\mathrm{V}_{1}\left(\mathrm{I}_{2}\right.$ при $\left.k=\frac{2 n+1}{3}, \mathrm{~V}_{1}, \mathrm{VI}_{3}\right)$. 
Размерность пространства тензоров $c_{j q}^{i}\left(c_{i j q}\right)$, инвариантных относительно $\Phi(H)$ $u \Psi(G)$, уменьиается на 1 при расширении $H$ до группь $G u$

а) уменьшается с 1 до 0 для включений $\mathrm{I}_{1}$ при четных $k, \mathrm{I}_{2}$ nри четных $k$ u $k \geqslant$ $\frac{2 n+1}{3}, \mathrm{I}_{3}, \mathrm{I}_{4}$ при четных $k<\frac{2 n}{3}, \mathrm{I}_{7}$ при $n=3, \mathrm{VI}_{3} \quad\left(\mathrm{I}_{1}\right.$ при четных $k, \mathrm{I}_{2}$ при четных $k$ и $k>\frac{2 n+1}{3}, \mathrm{I}_{3}, \mathrm{I}_{4}$ при четных $k<\frac{2 n}{3}, \mathrm{I}_{6}$ при $n=5, \mathrm{I}_{7}$ при $\left.n=3, \mathrm{I}_{10}\right)$;

б) уменьшается с 2 до 1 для включений $\mathrm{II}_{2}, \mathrm{II}_{4}, \mathrm{II}_{6} \quad\left(\mathrm{I}_{4} n p u k=\frac{2 n}{3}, \mathrm{I}_{6} n p u n=3\right.$, $\mathrm{I}_{7}$ nрu $\left.n=2, \mathrm{II}_{2}, \mathrm{II}_{4}, \mathrm{II}_{6}, \mathrm{VI}_{2}\right)$.

Размерность пространства тензоров $c_{j q}^{i}\left(c_{i j q}\right)$, инвариантных относительно $\Phi(H)$ и $\Psi(G)$, уменьиается на 2 при расширении $H$ до группь $G$ и

а) уменьшается с 2 до 0 для включений $\mathrm{I}_{4}$ при четных $k \geqslant \frac{2 n}{3}, \mathrm{I}_{5}$ при нечетных $n$, $\mathrm{I}_{6}$ при $n=3, \mathrm{I}_{11}, \mathrm{IV}_{7}, \mathrm{VI}_{2} \quad\left(\mathrm{I}_{4}\right.$ при четных $k>\frac{2 n}{3}, \mathrm{I}_{5}$ при нечетных $\left.n, \mathrm{I}_{11}, \mathrm{IV}_{7}\right)$;

б) уменьшается с 3 до 1 для включений $\mathrm{II}_{1} \quad\left(\mathrm{II}_{1}, \mathrm{VI}_{1}\right)$.

Размерность пространства тензоров $c_{j q}^{i}$, инвариантных относительно $\Phi(H)$ и $\Psi(G)$, уменьшается с 3 до 0 при расширении $H$ до группы $G$ для включения $\mathrm{VI}_{1}$.

Размерность пространства тензоров $c_{j q}^{i}\left(c_{i q j}\right)$, инвариантных относительно $\Phi(H)$ и $\Psi(G)$, уменьшается на 6 при расширении $H$ до группь $G$ и

а) изменяется с 6 до 0 для включения $\left(\mathrm{I}_{12}\right)$;

б) уменьиается с 7 до 1 для включений $\mathrm{II}_{8} \quad\left(\mathrm{II}_{8}\right)$.

Размерность пространства тензоров $c_{j q}^{i}\left(c_{i j q}\right)$, инвариантных относительно $\Phi(H)$ и $\Psi(G)$, уменьшается $с 10$ до 0 при расширении $H$ до группь $G$ для включений $\operatorname{IV}_{10}$ $\left(\mathrm{IV}_{10}\right)$.

Размерность пространства тензоров $c_{j q}^{i}\left(c_{i j q}\right)$, инвариантных относительно $\Phi(H)$ $u \Psi(G)$, при расширении $H$ до группь $G$ для включения $\mathrm{III}_{1}$ уменьшается $c\left[\frac{k}{2}\right]+1$ до 1 при четных $k$ и до 0 при нечетньх $k$ вне зависимости от типа тензора.

\section{ЗАМЕЧАНИЯ.}

1. В теореме сохранена нумерация включений таблицы 5 [3].

2. Переменные $k$ и $n$ принимают натуральные значения.

3. Из-за очень больших трудностей в разложении тензорного произведения на неприводимые нами не рассмотрено включение $\mathrm{IV}_{1}$.

4. Результаты для тензоров $c_{i j q}$ даны в круглых скобках.

\section{СПИСОК ЛИТЕРАТУРЫ}

[1] Lie S. Theorie der Transformationsgruppen. B. 3. Leipzig, 1893. [2] Морозов B. B. // Матем. сб. 1939. Т. 5 (47). С. 355-390. [3] Дынкин Е. Б. // Труды ММО. 1952. Т. 1. С. 39-166. [4] Мантуров О. В. // ДАН СССР. 1985. Т. 281. С. 1048-1051.

Поморский международный педагогический университет им. М.В. Ломоносова
Принято редколлегией 11.03.1996 